\section{MEHR TÖDLICHE INFEKTIONEN}

\section{C. difficile wird gefährlicher}

Inzidenz und Schweregrad von Clostridiumdifficile-Infektionen (CDAD) nehmen zu. Schuld könnte das Auftreten von hypervirulenten Stämmen sein, ergab eine Studie an der Uniklinik Tampere. Von insgesamt 780 CDADFällen gingen 111 (14,2\%) auf hypervirulente Stämme zurück. Die 30-Tage-Mortalität dieser Infekte betrug $20,7 \%$ gegenüber $8,5 \%$ bei
CDAD, die durch andere Stämme ausgelöst waren. Einen Hinweis auf den infizierenden C.-difficile-Stamm und damit auf die Prognose können die Leukozyten liefern. Patienten mit einem hypervirulenten Typ hatten signifikant höhere Werte (11,0 vs. 9,4 x 109/I).

International Journal of Infectious Diseases 2012; online 7 May 2012

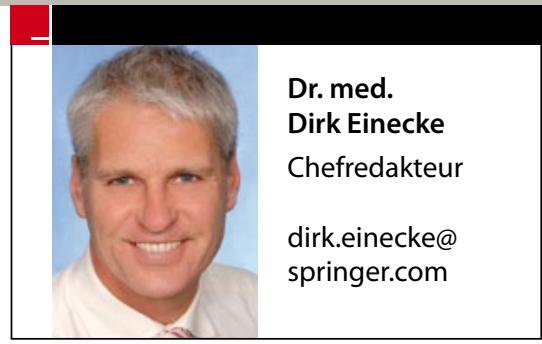

WENIGER PSYCHISCHE PROBLEME

\title{
Viel Bewegung ist gut für Kinderseelen
}

\section{HEILSAME WIRKUNG}

\section{Wallfahrt für gutes Cholesterin}

Wer sich auf Wallfahrt begibt und zügig seinem Ziel zupilgert, tut auch segensreiches für seinen Cholesterinspiegel. Schnell ausschreitende Pilger dürfen sich einen stärkeren Anstieg ihres HDL-Cholesterins erhoffen als Wegbegleiter, die gemächlicher unterwegs sind. Der Lohn der Langsamkeit ist dafür eine deutlichere Absenkung des LDL-Cholesterin-Spiegels. Das hat eine Studie ergeben, für die 29 gesunde Personen $280 \mathrm{~km}$ auf dem Pilgerpfad nach Santiago de Compostela zurücklegten. Die schnellere Gruppe $(n=15)$ mit einer Durchschnittsgeschwindigkeit von $4,6 \mathrm{~km} / \mathrm{h}$, die langsamere

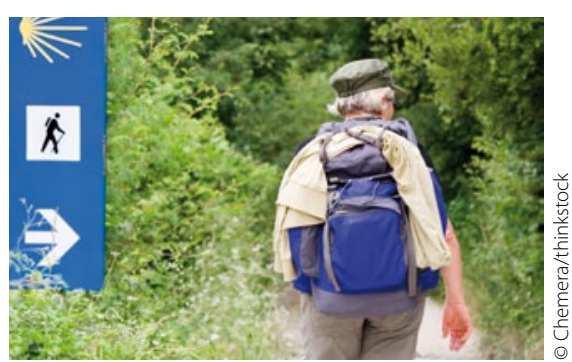

Wallfahrt für Herz und Seele.

mit 4,1 km/h. Jeden zweiten Tag waren Blutproben genommen worden.

BMJ Open 2012;2:e000875. doi:10.1136/ bmjopen-2012-000875
Kinder, die ausgiebig körperlich aktiv sind, werden deutlich seltener sozial oder emotional auffällig. Auch leiden sie weniger an Ängsten und Depressionen. Finnische Forscher aus Helsinki und Oulu hatten für ihre Studie rund 200 achtjährige Kinder mit Beschleunigungssensoren an den Handgelenken ausgerüstet. Verglichen wurden die psychischen Merkmale der aktivsten mit den am wenigsten aktiven Kinder. Nach Angaben von Müttern und Lehrern waren die aktiven Kinder offenbar auch glücklicher und ausgeglichener, seltener ängstlich oder depressiv, hatten weniger Aufmerksamkeitsprobleme und neigten nicht zu Trotzreaktionen und Aggressivität.

J Pediatr 2012; DOl: http://dx.doi.org/10.1016/j. jpeds.2012.03.037

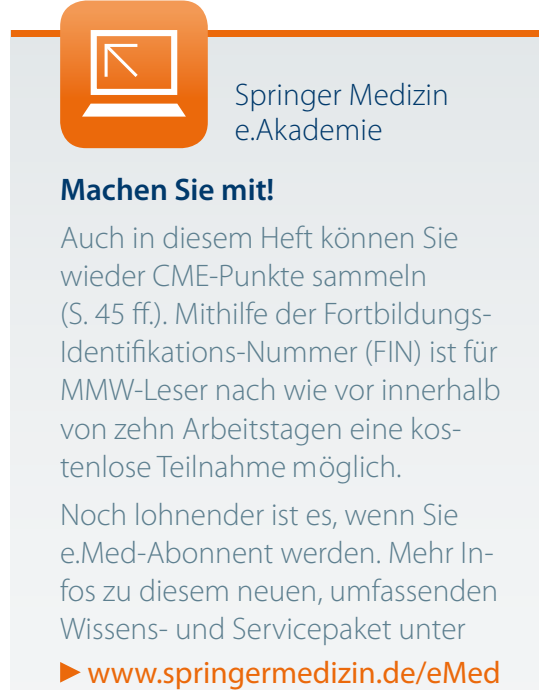

\section{FUNKTIONSTESTS ENTSCHEIDEND}

\section{Mit dem Knieersatz nicht zu lange warten!}

Möglicherweise warten viele Patienten mit Kniegelenksarthrose zu lange, bis sie sich zu einem Kunstknie durchringen. Die Patienten passen ihre Aktivität zunehmend den Schmerzen an, die Folge ist ein Verlust an Muskelmasse, Kraft und Funktionalität. Dies wiederum sind schlechte Voraussetzungen für das funktionelle Ergebnis einer Arthroplastie. In einer US-amerikanischen Studie mit 119 Knieersatzpatienten schnitten postoperativ diejenigen am schlechtesten ab, die z.B. im "Timed-Up-and-Go-Test" (TUG) vor dem Eingriff mehr als 10 Sekunden gebraucht hatten, um von einem Stuhl aufzustehen, drei Meter zu gehen und sich wieder hinzusetzen. Ähnlich aussagekräftig waren der "Six-MinuteWalk" (6MW) sowie der Stair-Climbing-Test" (SCT).

Aus den Ergebnissen dieser Tests und den Antworten auf einen Gesundheitsfragebogen mit physischer (PCS) und psychischer (MCS) Komponente könne man die Funktionalität prognostizieren, die nach Kniegelenksersatz erwartet werden kann.

J Orthop Res, published online 26 Apr 2012; DOI 10.1002/jor.22140 